\title{
Detection of significant left renal artery stenosis caused by fibromuscular dysplasia with selective angiography
}

\author{
Ramezan Jafari ${ }^{1}$, Zohreh Rostami ${ }^{2}$, Mohammad Nikpoor ${ }^{2}$, Mohammad Javanbakht ${ }^{2}$, \\ Mohsen Sadeghi Ghahroudi ${ }^{3}$, Mahbobeh Sadat Hosseini ${ }^{4}$, Behzad Einollahi ${ }^{2}$ \\ ${ }^{1}$ Department of Radiology and Chemical Injuries Research Center, Baqiyatallah University of Medical Sciences, Tehran, Iran; \\ ${ }^{2}$ Nephrology and Urology Research Center, Baqiyatallah University of Medical Sciences, Tehran, Iran; \\ ${ }^{3}$ Atherosclerosis Research Center, Baqiyatallah University of Medical Sciences, Tehran, Iran; \\ ${ }^{4}$ Clinical Research Development Unit, Baqiyatallah University of Medical Sciences, Tehran, Iran.
}

\begin{abstract}
Summary A 22-year-old female, was referred with a history of a headache and elevated blood pressure without family history of hypertension or familial dyslipidemia. Initially, a spiral computed tomographic angiography of the renal arteries was conducted, demonstrating completely abnormal left renal artery at the medial portion of the vessel with suspicious stenosis, which was supposed to be due to fibromuscular dysplasia (FMD). Subsequently, the patient underwent selective renal angiography and balloon angioplasty. Severe stenosis was observed on the left side and moderate stenosis on the right side in the medial and proximal part of the vessels, respectively. After the diagnosis of FMD, the left side was treated by balloon and finally, the patient was discharged with good control of blood pressure by losartan/amlodipine treatment.
\end{abstract}

KEY WORDS: Fibromuscular dysplasia; Computed tomographic angiography; Selective renal angiography; Balloon.

Submitted 10 August 2019; Accepted 16 October 2019

\section{INTRODUCTION}

Fibromuscular dysplasia (FMD) with renovascular hypertension (RVH) is idiopathic with unclear etiology, being a non-inflammatory and non-atherosclerotic vascular disease, which mainly involves the renal arteries leading to RVH. It less commonly affects carotids, vertebral, iliac, subclavian, and visceral arteries $(1,2)$.

FMD is mainly observed in young women and only $10 \%$ to $20 \%$ of the cases present with renal artery stenosis (RAS), causing secondary RVH. The right RA is the prevailing site of FMD although bilateral manifestation is possible in $40 \%$ of cases and approximately bilateral FMD is seen in one-third of cases and unilateral FMD of the right RA is three times higher than FMD of the left RA $(3,4)$.

Invasive selective renal artery angiography procedure has been considered as the gold standard for the detection and assessment of RAS caused by FMD although noninvasive diagnostic techniques such as color Doppler ultrasonography and CT angiography can discover RAS, especially when localized near to the vascular origin (5, 6). In present study, we report a 22 -year-old female with a history of a headache and hypertension due to RAS caused by FMD who was successfully treated.

\section{Case presentation}

A 22-year-old female patient was referred to our clinic with a history of a headache and elevated blood pressure. After primary examination and relative blood pressure control (from 190/120 to $160 / 100 \mathrm{mmHg}$ ) she was hospitalized for further investigation in the hospital. Additionally, she had with no family history of hypertension or familial dyslipidemia and no history of cigarette smoking, alcohol and drug consumption. On physical examination, the patient had normal jugular venous pressure. Moreover, in her cardiac exam, there was a systolic murmur (3/6) at the apex. No bruits were heard on her abdomen and carotid regions, and also, the upper and lower extremities pulse was normal and symmetrical. Her laboratory results included: WBC: 4.600 Neutrophils 71\%, Hemoglobin: 9.3 g/dl, MCV: 87 fl, MCH: 29 pg, Platelet: 360000, Liver function tests: normal, Erythrocyte sedimentation rate: $8 \mathrm{~mm} / \mathrm{h}, C$ reactive protein: negative, Antinuclear antibody: negative, Rheumatic factor: negative, Anti dsDNA antibody: negative, C3: normal, C4: normal, Calcium: normal, Magnesium: normal, Creatinine: 0.8 mg/dl, Sodium: $136 \mathrm{mEq} / \mathrm{dl}$, Potassium: $3.7 \mathrm{mEq} / \mathrm{dl}, 24 \mathrm{hr}$ urine protein: normal, Aldosterone: $1230 \mathrm{ng} / \mathrm{dl}$, Plasma Renin Activity: $61.5 \mathrm{ng} / \mathrm{ml}$.

At transthoracic echocardiography investigation, she had a mild enlarged left ventricular size and normal function (moderate left ventricular hypertrophy), normal right ventricle size and function, moderate to severe mitral regurgitation, mild to moderate tricuspid regurgitation, moderate aorta insufficiency.

Renal ultrasonography revealed right and left kidney measuring 107 and $90 \mathrm{~mm}$ in length, respectively. Color Doppler ultrasound indicated that velocity at the origin of the right renal artery was $359 \mathrm{~cm} / \mathrm{s}$ and at the left was $95 \mathrm{~cm} / \mathrm{s}$ with a resistive index (RI) of 0.60-0.62 and 0.390.48 at the right and left renal artery, respectively. In consideration of the size difference of the two kidneys and of the reduced RI, plus the elevated serum levels of renin and aldosterone, a supplementary study with computed tomographic angiography (CTA) was recommended.

A spiral CTA of the renal arteries was conducted, revealing unilateral renal FMD. The left renal artery was completely abnormal at the medial portion of the vessel with suspicious stenosis, which was related to FMD at that 
Figure 1.

Axial (A) coronal (B) and Volume Rendering Technique (VRT) of computed tomography (CT) scan images showing beading at mid and distal third of left renal artery with significant stenosis at midportion and sparing of origin which is compatible with Fibromuscular dysplasia (FMD).

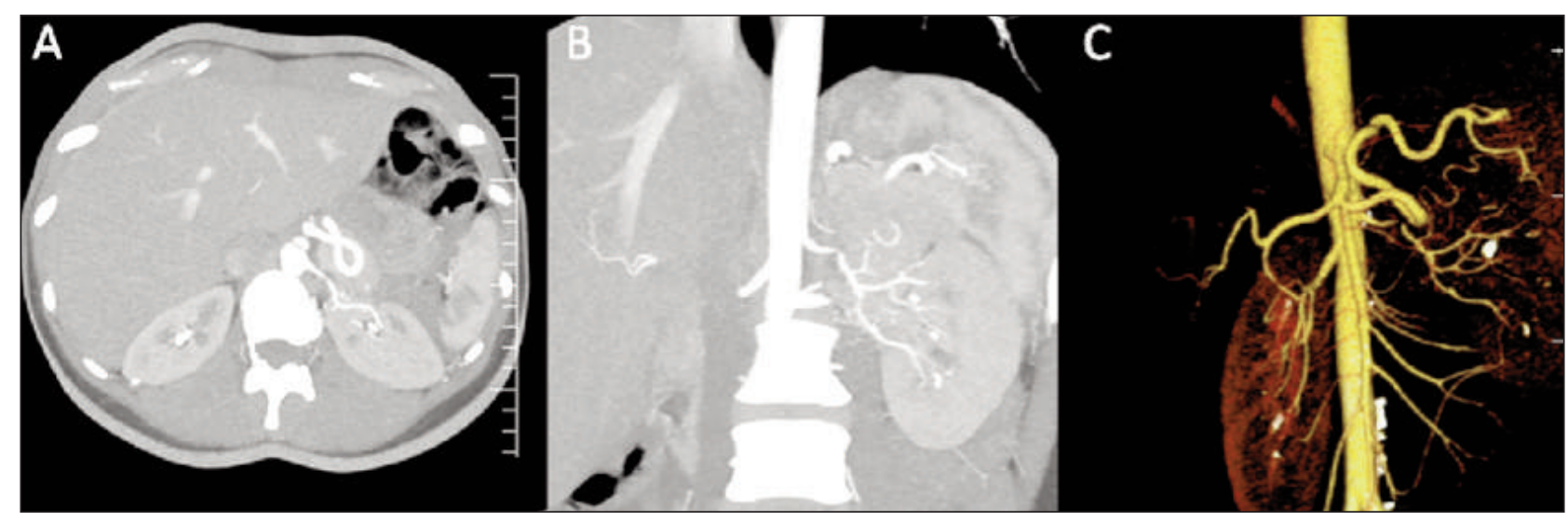

time. A selective renal angiography was performed, demonstrating patent right renal artery without stenosis (Figire 1) and mild beading of branches of left renal artery branches. Additionally, no accessory or aberrant arterial branch was detected.

She underwent selective renal angiography and balloon angioplasty. The most critical stenosis was on the left side (severe) but moderate stenosis was also seen on the right side in the proximal part of the vessel.

The left side (more severely affected by FMD) was treated by balloon on the medium part of the vessel. There was a good angiographic outcome after this approach: the stenosis ameliorated notably and there were no spasms or dissections. There were no complications. After angioplasty, she was discharged with good control of blood pressure with losartan/amlodipine therapy. She was asymptomatic and in good overall clinical condition and well-controlled blood pressure was observed after discharge.

\section{Discussion}

FMD as an unusual cause of arterial disease, which mainly affects females (aged 15-50 years), and regularly involves the mid or/and distal segments of the renal artery $(4,7)$. In accordance, our case was a 22 -year-old female with a stenosis of the renal artery at the medial portion of the vessel.

On the other hand, notwithstanding diverse theories involving genetic, mechanical and hormonal risk factors, cigarette smoking, cardiovascular risk factors and also, intrinsic deficiency of elastic fibers, the pathogenesis of FMD remains undisclosed $(8,9)$.

Medical management and pharmacological therapy of hypertension in FMD should pursue the guidelines of the Joint National Committee on interdiction, inspection, assessment and remedy of high blood pressure (10). The first line therapy when managing patients with symptomatic FMD is the treatment of blood pressure that in patients with RAS needs at least one antihypertensive drug (11). Furthermore, in young patients, revascularization is an alternative in cases with hypertension refractory to pharmacological therapy (12). Balloon angioplasty is beneficial for the remedy of FMD in the principal renal arteries. However, nowadays it is possible to use smaller balloons and better catheter techniques $(13,14)$. In accordance with the above reported treatment approaches, our patient affected by FMD was treated by balloon on the medium part of the left renal vessel and was discharged with control of blood pressure using losartan/amlodipine. Finally, subsequent the angioplasty, the blood pressure in our case returned to normal on antihypertensive drugs.

\section{Conclusions}

FMD causing RAS and renovascular hypertension is fundamental to be considered in young hypertensives, even in absence of family history of hypertension. Moreover, balloon angioplasty as well as selective renal angiography is the 'gold standard' test for RAS and must be conducted when renovascular intervention is envisaged.

\section{Consent}

Informed patient consent was obtained.

\section{REFERENCES}

1. Geavlete O, Calin C, Croitoru M, et al. Fibromuscular dysplasia - a rare cause of renovascular hypertension. Case study and overview of the literature data. J Med Life. 2012; 5:316-20.

2. Hundae AY, Hebert CA, Schussler JM. Fibromuscular dysplasia of the renal artery as a cause of secondary hypertension. Proc (Bayl Univ Med Cent). 2013; 26:405-6.

3. Chrysant SG, Chrysant GS. Treatment of hypertension in patients with renal artery stenosis due to fibromuscular dysplasia of the renal arteries. Cardiovasc Diagn Ther. 2014; 4:36-43.

4. Zeina AR, Vladimir W, Barmeir E. Fibromuscular dysplasia in an accessory renal artery causing renovascular hypertension: a case report. J Med Case Rep. 2007; 1:58.

5. Colyer WR, Eltahawy E, Cooper CJ. Renal artery stenosis: optimizing diagnosis and treatment. Prog Cardiovasc Dis. 2011; 54:29-35. 
6. Radermacher J, Chavan A, Schäffer J, et al. Detection of significant renal artery stenosis with color Doppler sonography: combining extrarenal and intrarenal approaches to minimize technical failure. Clin Nephrol. 2000; 53:333-43.

7. Urban BA, Ratner LE, Fishman EK. Three-dimensional Volumerendered CT angiography of the renal arteries and veins: normal anatomy, variants, and clinical applications. Radiographics. 2001; 21:373-386.

8. Slovut DP, Olin JW. Fibromusculardysplasia. N Engl J Med. 2004; 50:1862-1871.

9. Ralapanawa DM, Jayawickreme KP, Ekanayake EM. A case of treatable hypertension: fibromuscular dysplasia of renal arteries. BMC Res Notes. 2016; 9:6.

10. Chobanian AV, Bakris GL, Black HR, et al. The seventh report of the Joint National Committee on Prevention, detection, evaluation, and treatment of high blood pressure: the JNC 7 report. JAMA. 2003; 289:2560-2572.
11. Weinberg I, Gu X, Giri J, et al. Anti-platelet and anti-hypertension medication use in patients with fibromuscular dysplasia: results from the United States Registry for fibromuscular dysplasia. Vasc Med. 2015; 20:447-453.

12. Knorring JV, Edgren J, Lepäntalo M. Long-term results of percutaneous transluminal angioplasty in renovascular hypertension. Acta Radiologica. 1996; 37:36-40.

13. Gumus B, Cevik H, Vuran C, et al. Cutting balloon angioplasty of bilateral renal artery stenosis due to Takayasu arteritis in a 5year-old child with midterm follow-up. Cardiovasc Intervent Radiol. 2010; 33:394-7.

14. Alhadad A, Mattiasson I, Ivancev K, et al. Revascularisation of renal artery stenosis caused by fibromuscular dysplasia: effects on blood pressure during 7-year follow-up are influenced by duration of hypertension and branch artery stenosis. J Hum Hypertens. 2005; 19:761-7.

\section{Correspondence}

\section{Ramezan Jafari, MD}

Department of Radiology and Chemical Injuries Research Center,

Baqiyatallah University of Medical Sciences, Tehran (Iran)

Mohammad Javanbakht, MD (Corresponding Author)

mhmjvbt81@gmail.com

Zohreh Rostami, MD

Mohammad Nikpoor, MD

Behzad Einollahi, MD

Nephrology and Urology Research Center, Baqiyatallah University of Medical Sciences, Tehran (Iran)

Mohsen Sadeghi Ghahroudi, MD

Atherosclerosis Research Center, Baqiyatallah University of Medical Sciences, Tehran (Iran)

Mahbobeh Sadat Hosseini, MD

Clinical Research Development Unit, Baqiyatallah University of Medical

Sciences, Tehran (Iran) 